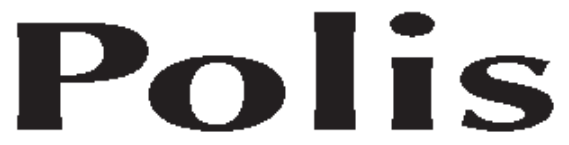

\title{
La Conservación Integral Alternativa desde el Sur: una visión diferente de la conservación
}

\author{
Isa Torrealba* \\ Fabricio Carbonell**
}

Resumen: Esbozamos los elementos de un nuevo modelo conceptual para entender desde la perspectiva latinoamericana qué ocasiona el desbalance entre naturaleza y sociedad y vislumbrar así las verdaderas oportunidades y amenazas de soslayar el abismo entre lo ambiental y lo social al integrar efectivamente desarrollo y conservación. Como propuesta teórica endógena compatible con la perdurabilidad del bienestar humano y producto de 10 años de investigación en el medio rural latinoamericano (sur), presentamos al enfoque Conservación Integral Alternativa. En este artículo además de considerar los temas de pobreza, población y neoliberalismo, entre otros, redefinimos los conceptos de desarrollo sustentable, diversidad biocultural y conservación biológica; los que, analizados a la luz de valores ancestrales, acumulación de riqueza, agricultura para la vida y políticas ambientales, arrojan un panorama diferente de la visión convencional de la conservación.

Palabras clave: conservación, desarrollo, sustentabilidad, transdisciplinariedad.

\section{The Alternative Integral Conservation from the South: a different view about conservation}

Abstract: After 10 years of effort as practitioners in Latin American rural areas, and as an endogenous proposal, where sustainability and human well-being are matched, we introduce the CIA-Sur (Alternative Integral Conservation from the South) approach. We sketch the elements of this conceptual model in order to understand, from the Latin American standpoint, what is causing the imbalance between nature and society. We sought to find the actual opportunities and threats of overcoming the environmental and social abyss that emerges when we put together development and conservation. We also redefine here the following terms: Sustainable development, bio-cultural diversity, biological conservation, richness accumulation, life agriculture and environmental policies, in order to find a non conventional vision of future conservationists styles in our region.

\footnotetext{
* ONG Meralvis, Heredia, Costa Rica. Email: itorrealNOSPAM@yahoo.com; itorreal@yahoo.com

** ONG Meralvis, Heredia, Costa Rica. Email: carbon_f@yahoo.com.mx; ong_meralvis@yahoo.com 
Key-words: conservation, development, sustainability, trans-disciplines.

\section{Introducción}

Al nivel mundial, pese los esfuerzos hechos vía políticas y legislación y los avances tenidos en cuanto a mejoras en la salud y mayor producción de alimentos, los problemas ambientales y sociales se incrementaron contundentemente desde la segunda mitad del siglo XX. Desde fines del siglo XX, la brecha entre ricos y pobres incrementó en muchas regiones del mundo y hoy se habla de un tercer mundo en el primer mundo y viceversa (Hinkenlammert 1999). La revolución verde, ideada después de la 2da Guerra Mundial, aunque contribuyó a tener más alimentos, no mejoró la distribución de los mismos al tiempo que generó gran contaminación química; y los avances de la tecnología y la medicina, aunque generaron soluciones sanitarias, éstas no fueron accesibles a todos. En el fondo, prevaleció una visión ambiciosa de tener cada vez más, irrespetuosa con la naturaleza y con la gente rural, donde la naturaleza y los grupos sin poder subsidian el crecimiento de unos a costa de los otros. Hoy, uno de los principales problemas mundiales con la conservación de la biodiversidad y el desarrollo sustentable ${ }^{1}$ es que, por un lado, la conservación ha estado liderada por personas de marcada visión ambiental, a merced de los desarrollistas y por el otro, el desarrollo ha estado liderado por personas de marcado enfoque social, político y económico, a merced de los ambientalistas. Naturalistas, biólogos y forestales han estado, en cierta forma, regidos por las tendencias de los desarrollistas. A su vez, al éstos ver en la conservación un fin de mercado, han delegado en los ambientalistas el que ésta se afiance en zonas lejos de sus intereses tangibles inmediatos, sin percatarse de las implicaciones que sus decisiones tendrían en el futuro. Sin darse cuenta de la presión que ejercen por lo que predican, en los enfoques de unos y otros ha surgido una gran desintegración. En tal fragmentación han perdido de vista que lo uno está inmerso dentro de lo otro; así como los problemas de conservación son más que todo políticos, económicos, sociales y éticos (McNeely et al. 1990), los del desarrollo tienen raíces ambientales, biofísicas y naturales.

Dada tal división, dos enfoques típicos han sido priorizar "gente" o "natura" (Stem et al. 2005). Si se enfatiza la visión antropocéntrica, se considera a la conservación como un

\footnotetext{
${ }^{1}$ Si bien en Costa Rica se prefiere usar el término sostenibilidad, muchas veces éste es confundido con la posibilidad de un crecimiento ilimitado lo cual atenta contra los objetivos mismos de lo perdurable o "sostenible". El término sustentable parece más acorde con la idea de perdurabilidad de la humanidad sin la merma de nuestro capital natural, ni social; por eso escogimos éste.
} 
medio para lograr el fin de facilitar la viabilidad comunitaria. Si se enfatiza la visión biocéntrica, la meta es lograr la protección de espacios naturales buscando integrar los esfuerzos entre desarrollo y conservación (aunque en la práctica estos se mantengan separados); en este caso se usa al desarrollo como medio para lograr conservación. Si queremos integrar efectivamente conservación y desarrollo, resulta prioritario buscar cómo ponderar equitativamente ambos lados, gente y natura, especialmente en la región latinoamericana rica en ambos. Así, nuestra tendencia es ver ambiente y sociedad íntimamente relacionados, sin considerar sólo tendencias de ecosistemas, eco-regiones, de los estilos de desarrollo y de las economías, sino todas relacionadas en iguales condiciones.

Tras una gran praxis investigativa en el medio rural latinoamericano, llegamos a ver la importancia de tres puntos claves, de los cuales aquí esbozamos sólo el primero: 1ro) la urgida necesidad de desarrollar un nuevo modelo integrador del arte y las ciencias sociales con las naturales, para crear una forma nuevas de abordar los problemas propios de la conservación de la biodiversidad cultural dentro del paradigma del desarrollo sustentable; 2 do) el requisito vital de virar en $180^{\circ}$ el sistema económico y político actual dirigido al ecocidio y genocidio, por el de una economía sustentable con fundamento en un sistema político más justo -menos acumulativo y más equitativo- para que la humanidad perdure; y 3ro) la necesidad de ver que estamos ante un cambio de época; esta nueva era necesita que se mire al mundo de otro modo.

\section{Métodos y área de estudio}

En el transcurso de los últimos años de nuestro proceso investigativo en América Latina dentro del campo de la conservación vinculada a la gente (que para el 2008 data de 18 años), llegamos a entender que una de las principales causas de la disparidad entre conservación y desarrollo es que las políticas y visiones de desarrollo y conservación vienen dadas por agendas externas. De allí la necesidad de crear un nuevo modelo: una visión sistémica, integral, alternativa y endógena. Al partir de una visión de desarrollo compatible con la sustentabilidad y generadora de un beneficio humano integral, visionamos a las comunidades como actores claves en el mejoramiento de su ambiente y a sus pobladores rurales e indígenas como custodios y co-manejadores de áreas silvestres integrales alternativas (hoy llamadas parques o áreas protegidas), de sus otras zonas naturales y agro-paisajes; teniéndolas a todas como opción de uso con respeto para su beneficio integral. A tal visión la hemos denominado CIA-Sur, conservación integral alternativa desde Latinoamérica, desde el sur.

En la construcción de la CIA-Sur tomamos diez años de trayectoria en proyectos de investigación y extensión en Latinoamérica por parte de la ONG Meralvis (Mejorando al desarrollo rural regional a través de la conservación de la vida silvestre), la cual surgió para empoderar con enfoque de género a grupos no-dominantes, vía el facilitamiento e 
intercambio de información sobre aspectos sociales y tendencias actuales de conservación, biodiversidad y vida silvestre. Entre 1997 y el 2007 tuvimos acciones en cinco países con 26 proyectos de investigación y extensión donde enfocamos, en una mezcla de métodos biológicos y sociológicos, la concepción utilitaria de los recursos naturales para enriquecer al desarrollo rural; de éstos 14 fueron rigurosos estudios socio-ambientales (Cuadro 1). A lo largo de eventos de difusión de dichas investigaciones, vimos que nuestro enfoque no encajaba con las disciplinas tradicionales; aunque tuvimos apertura con los sociólogos, supimos que no calzábamos del todo con los enfoques de la sociología rural o de los recursos naturales (SRN), ni de la ambiental (SAM). ${ }^{2}$ En Meralvis nos interesan los valores $\mathrm{y}$ actitudes de la gente sobre el ambiente (de la SAM), en un marco que busca entender cómo el uso de los recursos naturales afecta a la gente (de la SRN); a su vez, tenemos una orientación macro en relación con las Agendas Globales del Desarrollo que intentan promover el desarrollo sustentable (de la SAM), pero partimos de un enfoque específico local de lugares y recursos en particular (de la SRN). Tras esta gran experiencia investigativa basada en campo, analizamos nuestra ejecutoria y vimos que éramos parte de un área a la que llamamos sociología de la conservación.

Cuadro 1. Catorce proyectos rigurosos con períodos promedio de 5 meses en campo (2,5-14 meses) entre 1997 -2007, en comunidades campesinas e indígenas.

\begin{tabular}{|c|c|c|c|}
\hline Países & Programa & Comunidades & $\begin{array}{l}\text { A grandes rasgos lo que estudiaron los } \\
\text { proyectos }\end{array}$ \\
\hline $\begin{array}{l}\text { Costa Rica, } \\
\text { Bolivia, Chile y } \\
\text { Perú }\end{array}$ & $\begin{array}{l}\text { INFOCON } \\
5 \text { Proyectos }\end{array}$ & $\begin{array}{l}\text { Indígenas Ngöbe, Bribri, } \\
\text { Cabécar, Huetar, Maleku, } \\
\text { Aymara }\end{array}$ & $\begin{array}{l}\text { Problemas por el uso de mamíferos silvestres } \\
\text { grandes en relación con su conservación. También } \\
\text { problemas por el uso de humedales altoandinos en } \\
\text { relación con la ganadería ovina y de camélidos. }\end{array}$ \\
\hline Costa Rica & $\begin{array}{l}(1997-2000) \\
\text { COSECOM } \\
6 \text { Proyectos }\end{array}$ & $\begin{array}{l}\text { Agricultores rurales (de } \\
\text { subsistencia). Campesinos } \\
\text { ganaderos. Criadores de } \\
\text { mariposas. }\end{array}$ & $\begin{array}{l}\text { Problemas por el uso de ciertos ecosistemas } \\
\text { (manglares y bosque primario) o de la fauna } \\
\text { silvestre, en relación con su producción en } \\
\text { zoocriaderos y su conservación. }\end{array}$ \\
\hline $\begin{array}{l}\text { Costa Rica y } \\
\text { Panamá }\end{array}$ & $\begin{array}{l}\text { (1997-2004) } \\
\text { INTEGRAL } \\
3 \text { Proyectos } \\
(2005-7)\end{array}$ & $\begin{array}{l}\text { Indígenas Ngöbe, Bribri, } \\
\text { Cabécar, Naso-Teribe, } \\
\text { funcionarios del ambiente, } \\
\text { estudiantes, campesinos y } \\
\text { ganaderos de varios tipos. }\end{array}$ & $\begin{array}{l}\text { Problemas por el uso de mamíferos grandes } \\
\text { silvestres en relación con su conservación, especies } \\
\text { amenazadas como anfibios y }\end{array}$ \\
\hline
\end{tabular}

Para la construcción de los elementos de la CIA-Sur usamos elementos conceptuales de gran variedad de autores, ${ }^{3}$ los más relevantes (alfabéticamente): Friket Berkes, por su visión del conocimiento ligado a la acción en el desarrollo sustentable al nivel de las comunidades locales, Kenneth Burke, por su visión integral y holística de la socioecología ${ }^{4}$ Fritjof Capra, por su visión holística de la teoría de sistemas aplicada al

\footnotetext{
${ }^{2}$ Amplia información sobre el debate entre estos conceptos puede ser encontrada en Buttel (2002) y Field et al. (2002).

${ }^{3}$ Referencias de éstos están en Torrealba y Carbonell 2008 (en prensa)

${ }^{4}$ Por ejemplo, la agro-ecología y la tesis de Gaia, serían socio-ecología Burkeana. 
campo de la ecología; Jared Diamond y Charles Mann por su rescate histórico de las situaciones socio-ambientales vividas por culturas ancestrales y su visión de hacia donde podríamos llegar en el futuro; Carl Folke por su visión de la humanidad y la naturaleza como sistemas dinámicos en continua interacción y co-evolución; Paulo Freire, por su tesis de la liberación del oprimido; Bertus Haverkort, por su visión de la agro-ecología y alimentación del mundo en el futuro; Francois Houtart, por su percepción de la relación político-económica y relevancia del Neoliberalismo; James Lovelock por su tesis de Gaia, ${ }^{5}$ Marina Michaelidou por su visión de la interdependencia comunidades-ecosistemas, e Immanuel Wallerstein, por su tesis del sistema-mundo. Nuestro análisis fue enriquecido vía discusiones y coloquios en nuestras clases de enfoque ambiental y social al nivel universitario. Asimismo, la cosmovisión de las etnias Kogi, Bribri y Cabecar, influyó en nuestra construcción. Otras fuentes útiles fueron: Castro (2003), Clay (1988), Colchester (2005), Chacón (2003), Davis (2001), Fallas (2003), Fournier (2000), Leal y Bonilla (2005), Molina y Palmer (2000, 2005), Monge-Nájera (1999), Muro (2005), Novo (2005), Quiroga (2001) y Skutnabb et al. (2003); el Programa Estado de la Nación en Desarrollo Humano Sostenible, el Centro Centroamericano de la Población de la Universidad de Costa Rica y, en especial, algunos de nuestros proyectos (i. e. Torrealba y Carbonell 2002, Carbonell et al. 2003, Carbonell y Torrealba 2005).

Los proyectos empleados como marco para la construcción de la CIA-Sur estuvieron localizados en Costa Rica. Al comparar la situación de los mismos con otros proyectos de la región, vimos que pese sus particularidades, el país bien servía como reflejo de lo sucedido en América Latina. Adicionalmente, si bien vivimos en Costa Rica desde hace más de 10 años, ambos autores somos suramericanos y tuvimos experiencias previas en nuestros países antes de venir a especializarnos aquí. Por tanto, cabe especificar el contexto nacional costarricense. En cuanto a conservación y desarrollo para Costa Rica, Centroamérica, el país pareciera poseer características que le permitirían acercarse hacia un genuino desarrollo rural sustentable y una efectiva conservación de la biodiversidad. Actualmente, el país posee índices de desarrollo superiores a los de sus homólogos del istmo, tales como una mayor esperanza de vida al nacer y un menor índice de mortalidad infantil (PEN 2004), sigue una política nacional gubernamental de desarrollo sustentable desde los 1990s, ha tenido recursos económicos fluidos de diversas fuentes -tanto para conservación, como para desarrollo- y funge líder internacional en aspectos ambientales. Sin embargo, todavía Costa Rica no alcanza la sustentabilidad y su biodiversidad continúa mermándose dado que muchas de sus áreas silvestres protegidas -bastión de batalla pro biodiversidad- confrontan innumerables problemas que atentan contra los objetivos de su creación. Fuera de los parques, la naturaleza confronta una situación de suelos erosionados, contaminación hídrica y atmosférica y sobrepoblación urbana (Estado de la Nación en

${ }^{5}$ Palabra de origen griego que significa "madre tierra" y según la cual todo el planeta es un sistema viviente. 
Desarrollo Humano Sostenible 2006). A su vez, aunque sobrevivan -dentro y fuera de los parques- pequeños parches de zonas con una agricultura diferente (orgánica, ecológica, otras) y un ecoturismo genuino (rural comunitario, otros) como medio de conservar lo natural y obtener un desarrollo propio, la sustentabilidad -implementada a través de la conservación como negocio- no ha generado resultados de valor agregado al nivel rural.

\section{Elementos conceptuales para una nueva visión de la conservación}

1er Elemento: «desarrollo es conservación». Según nuestra concepción el desarrollo implica a la conservación y viceversa. En la visión de un nuevo desarrollo hemos rescatado la percepción de economistas del siglo XIX, quienes vislumbraron que la civilización industrial llegaría a un estado estacionario del capital y la riqueza, el cual sería un adelanto en relación con quienes defendían que las personas debían aplastar y avanzar, doblando sus medios por consumir cosas que producen poco o ningún placer, excepto como íconos de riqueza (Mill 1848). Tal estado estacionario implica la sustentabilidad. Según Naredo (1996) John Stuart Mill y otros economistas hablaron ya desde hace más de 100 años de un desarrollo sin crecimiento e indica que lo sucedido al término de sustentabilidad es una corrupción mental. Pese la gran cantidad de literatura escrita, todavía hoy hablar de desarrollo sustentable implica mucha ambiguiedad y polémica, dado que se le ve como opción de crecimiento ilimitado en un afán desmedido por acumular riqueza a costa de enormes costos socio-ambientales. Lo que debe perdurar no es el mercado ni la producción creciente, sino la red de la vida (Capra 2000).

Así, vemos al desarrollo no como un proceso de mero crecimiento, sino como un proceso de cambio en el cual las personas mejoran su calidad de vida y se hacen útiles a la sociedad en función de lo que sean y hagan. Una sociedad que sólo tuviera miembros útiles sería mucho más desarrollada. Entendemos al desarrollo sustentable como un proceso de cambio progresivo en la calidad de vida del ser humano -centro y sujeto primordial del desarrollo- por medio del crecimiento económico limitado, con equidad social intra- e intergeneracional y con medios de producción y patrones de consumo transformados en perdurables; una sociedad sustentada en el equilibrio ecológico como soporte vital de la región (modificado de ALIDES 1997). Tal estilo de desarrollo implica respeto a la diversidad étnica y cultural regional, nacional y local, en una visión pluricultural diversa, tal como lo es Latinoamérica, tanto en lo bio-físico, como en lo socio-cultural. Dicho estilo requiere del fortalecimiento y la participación ciudadana, para que convivan en paz y armonía con la naturaleza, de manera tal que no se comprometa y se garantice la calidad de vida para las futuras generaciones.

Para nosotros no se puede hablar de desarrollo sino hablamos de conservación. Aquí 
distinguimos los conceptos de preservación, protección y conservación que muchas veces son mal manejados. El primero se asocia con la idea de la exclusión de la intervención humana en la naturaleza y el último con un uso respetuoso, más eficiente y sostenido de los recursos naturales. La conservación tiene su origen en el cuido ancestral de los recursos naturales; la preservación, fue expresada en la creación de parques y áreas protegidas en el siglo XX (Palacio 2001). Así, la protección implica un uso controlado. La preservación y la protección, tienen una base filosófica composicionalista, donde el ser humano se considera diferente a la naturaleza, mientras que nuestra visión de la conservación es más funcionalista, donde el ser humano es parte de los sistemas ecológicos. ${ }^{6}$ La conservación se compone de sistemas sociales y económicos, ecológicos y biológicos, donde la diversidad cultural-biológica es la base de su complejidad e interrelación con un ambiente dinámico y cambiante.

Para 1978 Michael Soulé definió la conservación biológica como metadisciplina, holística y multi-disciplinaria, orientada a promover esfuerzos para conservar y mantener la biodiversidad del planeta a través de la investigación, la educación, la comunicación y otros aspectos aplicados (Soulé 1986). Actualmente, ya no sería una metadisciplina o puente entre dos o más disciplinas, sino una ciencia aparte, que mantiene su condición holística mas no es exclusivamente multidisciplinaria, abundan los enfoques integrados interdisciplinarios, mientras que los intentos de fusión entre varias disciplinas o transdisciplinarios, ya se vislumbran. Debe ser transdisciplinaria en el sentido de fusionar distintas disciplinas porque sino perdería su razón de ser.

2do Elemento: «la diversidad biológico-cultural sustenta la vida ». Para nosotros la diversidad implica dos aspectos de un mismo continuum, en un lado está lo social y en el otro lo biológico. Al igual que economistas, políticos y desarrollistas en la parte de desarrollo sustentable han corrompido el significado propio de tal término; biólogos, forestales y ambientalistas hemos corrompido a la biodiversidad al enfatizar inapropiada y excesivamente su parte biológica en relación con la dimensión humana que le afecta y modifica. La diversidad bio-cultural comprende la diversidad lingüística, étnica y biológica vinculada con el lenguaje, el conocimiento y el ambiente (Dankelman y Ramprasad 2000, Skutnabb et al. 2003) y la biológica comprende genes, especies y ecosistemas (Botkin y Talbot 1992).

Si bien la biosfera comprende el menor porcentaje de superficie en comparación con los otros sistemas del planeta (hidrosfera, litosfera y atmósfera), la misma es decisiva para la vida en el mismo y tras años de procesos evolutivos hoy se encuentra vida diversa en las regiones geográficas más inhóspitas y extremas del mundo. La diversidad de especies del planeta (inter-específica) o su riqueza natural, se estima conservadoramente entre 14 y

${ }^{6}$ Para ampliar las bases filosóficas de la conservación, funcionalismo y composicionalismo, revísese Callicot et al. (1990) 
17.000.000 de especies biológicas discretas, de las cuales sólo se han descrito unos 2.000.000 (www.unep-wcmc.org/species/dbases/about.cfm). Todavía faltan muchas especies por describir; por ejemplo, sólo para Costa Rica entre 1995 y el 2004 (10 años) se describieron en promedio 3 especies nuevas por día gracias al trabajo sistemático del Instituto Nacional de Biodiversidad (Obando 2004). Al nivel mundial, la mayoría de las especies descritas son invertebrados, los cuales representan un $74 \%$; las plantas y algas representan alrededor de un $16 \%$. Los vertebrados que comprenden apenas el 2,7\%, son cruciales por su mayor complejidad y enorme diversidad genética en cada especie (intraespecífica). La diversidad entre paisajes se ve vía los ecosistemas, de los cuales se han descrito al menos 200 zonas de importancia crítica para la humanidad; pero, la diversidad simple es mucho mayor; por ejemplo sólo Costa Rica posee al menos 20 zonas de vida o diferentes tipos de ecosistemas vegetacionales al nivel ístmico, si se incluye la parte marina, la diversidad es aún mayor.

En cuanto a la diversidad cultural mundial existen todavía unas 6.000 lenguas y más de 400 grupos étnicos (www.rsnz.org/advisory/biodiversity/biocult_diversity.pdf). Si matemáticamente combinamos los múltiples usos, creencias, valores y conocimientos asociados a los recursos naturales por grupos y zonas, el número seguramente está por encima de la potencia 10. Vale recalcar, sin embargo, que el concepto original de biodiversidad incluye el aporte cultural de los pueblos; sería imposible imaginar la biodiversidad sin vincularla a su contexto espacio-temporal en particular y sin el aporte de las diversas culturas humanas que la han mantenido, enriquecido y contribuido a su cambio a través del flujo e intercambio de recursos genéticos y el conocimiento asociado a éste (Solís 2001). Cultura y natura se unen en cuanto a diversidad. Tal como se acaban tipos de animales y plantas en el transcurso de esta sexta extinción masiva de especies, también están en riesgo muchas lenguas y grupos étnicos. Hoy, existe gran consenso científico en que toda esta diversidad es importante para la continuidad de la vida en el planeta, con nosotros en ella.

3er elemento «hay sistemas complejos de interacciones múltiples que trascienden el nivel de ecosistemas e implican aprender a ver al mundo desde otra perspectiva». Desde nuestro punto de vista la conservación es uno de tales sistemas. La conservación como ciencia derivó de la ecología aplicada que implica la noción de (eco) sistemas y, por tanto, la inclusión de los seres humanos y sus efectos. Cada ecosistema es una comunidad de organismos vivos y su ambiente no vivo que interactúa como una unidad funcional entera donde todos sus miembros dependen unos de otros. En tal comunidad sus miembros, están todos interconectados por una vasta red de interrelaciones, no hay desechos, todo circula continuamente en la red de la vida y todo proviene de la energía solar. La vida que sustenta los ecosistemas surgió por cooperación, asociación y trabajo en redes. Como las acciones de conservación ocurren en una compleja dimensión humana que afecta a tal red de interrelaciones en los sistemas vivientes y no vivientes del planeta, al trascender el nivel de ecosistemas debemos, por fuerza, incluir variables sociales, económicas, políticas y culturales. 
Entre las razones de la pérdida de diversidad actual, está la carencia de una visión de la misma como un súper sistema socio-ambiental de elevada complejidad formado por múltiples variables. Los sistemas complejos son realidades únicas que operan impredeciblemente sin el amparo de una ciencia linear «causa-efecto»; al ser altamente dinámicos y cambiantes, su condición de estabilidad puede pasar de estable a turbulenta y si su resiliencia o la capacidad que tiene un ecosistema saludable de volver al funcionamiento normal tras una perturbación, es insuficiente, pueden cruzar un umbral generador de una sobre-presión local desencadenante de una serie de resultados en cascada, algunos irreversibles (Farrell y Twining-Ward 2005). Estos sistemas incluyen múltiples redes, donde interesa más la forma y el patrón derivado de las conexiones e interrelaciones, que la cantidad (medidas) o su constitución (materiales). La teoría de la complejidad que se ocupa del estudio de sistemas complejos, se basa en el estudio de patrones (Capra 2000). Hay sistemas complejos adaptativos que pueden evolucionar y aprender; tal es el caso de los ecosistemas y de los sistemas sociales humanos comprendidos dentro de nuestra visión de la conservación.

Por ello, debemos aprender a ver y buscar maneras de visualizar los patrones subyacentes, las redes de organización y el orden dentro del caos que están dados por las interrelaciones, y su calidad. El ver tales patrones depende del paradigma de ciencia que sigamos (Van Eijk 1999). Jürgen Habermas describió tres niveles de ciencia: El nivel empírico-analítico, dentro del paradigma positivista uni o multi-disciplinario, pretende la transferencia de tecnología, datos e información; es cuantitativo y sistemático en lugar de ser holista y es común en las ciencias naturales que aplican el método científico. Su intención es encontrar al conocimiento. El histórico-hermeneútico, dentro del paradigma constructivista inter-disciplinario, pretende un aprendizaje participativo; es más cualitativo y holístico que el previo y es común en los estudios híbridos entre las ciencias naturales y sociales, que aplican métodos como el de la investigación-acción. Su intención es construir el conocimiento. El crítico-emancipatorio, dentro del paradigma trascendentalista, pretende la transformación; es crítico, cualitativo y holístico y está presente en los estudios inter y transdisciplinarios, donde se busca una fusión de las ciencias y técnicas para un desarrollo consciente, usa métodos como el de aprendizaje-acción. Su intención es crear al conocimiento. Por tanto, según sea el nivel de ciencia enfocado, podremos visualizar distintos patrones, ya que es muy diferente si queremos encontrar, construir o crear al conocimiento.

Es por ello que debemos aprender a ver al mundo de otra manera, porque si lo seguimos viendo como una simple despensa de la cual extraer recursos ilimitadamente, incluyendo en el ambiente cosas que éste sólo puede procesar a cierto ritmo y en una cierta cantidad (cuando puede biodegradarlas); llegamos a una mezcla muy peligrosa. Hay ciclos o patrones que se repiten cada cierto número de años, siglos, que nuestra sociedad moderna aún no alcanza a visualizar por completo ni a respetar. Por ejemplo, las extinciones 
masivas y períodos de temperaturas más frías o más cálidas, además de grandes movimientos geológicos, suceden cada tanto hablando en largos períodos de tiempo. El problema no es que las especies se extingan, ni que la temperatura suba, es la frecuencia e intensidad a la cual la humanidad ha acelerado esos procesos, lo cual puede rebasar el umbral de resiliencia de los ecosistemas y generar condiciones altamente impredecibles y cambiantes, como para que el planeta continúe vivo de la manera que lo hemos conocido en los últimos milenios. Hay científicos que opinan ya estamos en el umbral de lo irreversible (Lovelock 2006).

4to elemento «hay instituciones sociales que pertenecen a grupos poderosos y actúan como fuerzas directrices afectando a los sistemas complejos». Las instituciones sociales no formales son las creencias y actitudes de las personas con relación a un problema dependiendo del paradigma seguido; no son organizaciones formales o del gobierno; incluyen muchas redes y conexiones intangibles (parentela, amistades), nociones de autoridad y liderazgo basadas en rasgos culturales más que en lo que signifiquen ciertos valores occidentales o ideas técnicas particulares (Visser 2003). Incluyen las normas y reglas sociales de las personas de una cultura y sociedad.Tales instituciones pertenecen a los «partícipes» que forman parte de un problema enfocado, dentro o alrededor del mismo (Woodhill 2004).

Dentro de los sistemas debemos entender que las instituciones están conectadas a las personas al nivel local, pero hay fuerzas en un panorama mayor al local. Tales fuerzas directrices están dadas por los paradigmas existentes en los grupos de personas y operan sobre y entre los diversos sistemas; se componen por las interrelaciones entre sus partícipes a los que les llegan vía la educación formal (escuela, colegio, universidad) e informal (tradición, amistad, extensión, medios de comunicación) (Scearce y Fulton 2004). Por ejemplo, una gran institución es la visión occidental europeizada del mundo y de la ciencia. Esta misma institución impidió que la sociedad con poder, viera que el Amazonas es un artefacto bio-cultural en lugar de una evolución natural prístina (Mann 2006). En ese caso el ser humano de hace siglos "construyó" tal ecosistema contribuyendo contundentemente al incremento de su riqueza natural, al tiempo que usaba sus recursos. Con los recursos de la información y la tecnología actuales, un ser humano más humanitario e inteligente que codicioso y avaro, bien habría estado construyendo al «destruir» desde hace rato. En lugar de eso, acaparamos biomasa, energía y mermamos nuestro capital natural planetario. Pero, no siempre ha sido así, ni tiene porqué continuar siéndolo. Aunque sólo seamos una especie, de nosotros depende que continúen aquí incluso hasta los 14-17 millones de especies en el planeta, piedras de base para la continuidad intra e inter-específica y paisajística de la vida; lo mismo que de la continuidad misma de nuestra especie con su bagaje de capital socio cultural. 


\section{Una nueva visión de la conservación: «la CIA-Sur»}

Para perdurar la diversidad biológica-cultural que nos sustenta no debe ser reducida a mercancía.

La verdad, es que el sistema de áreas silvestres protegidas tal como lo conocemos hoy en Latinoamérica, nunca se nos planteó como opción, sino que vino impuesto disimuladamente al amparo de premisas científicas incompletas y justificaciones sociales disfrazadas, dado que -por lo general- los y las profesionales unidisciplinarios -incluso en equipos multidisciplinarios y en algunos interdisciplinarios- veían únicamente una arista del problema y no la totalidad del mismo. Nunca hubo la opción de poder crear nuestro propio sistema de conservación, ni tampoco se supo la razón basal para necesitarlas. Por ello, es éticamente incorrecto no sólo culpar a las comunidades indígenas y rurales de la destrucción ambiental, sino pretender coartarles el uso de los recursos naturales que ellos cuidaron perdurablemente por centurias. Es incorrecto también querer aumentar a toda costa políticas y legislaciones proteccionistas "de papel", por cuanto cacería y pesca, entre otros tipos de usos de los recursos por parte de las comunidades rurales e indígenas, son factores culturales inmersos en las personas. Debemos informar, dar las herramientas y la capacitación correctas, para que la gente decida cómo y qué cambiar de cuáles prácticas en el uso de su biodiversidad.

Veamos el caso de Costa Rica. Sus Áreas Silvestres Protegidas (ASP) no poseen gran viabilidad ecológica por su pequeño tamaño, y muchas están en zonas "improductivas", como volcanes, humedales y áreas de gran pendiente; tampoco se ha probado que los enlaces biológicos puedan constituir una solución, como lo es el caso del Corredor Biológico Mesoamericano. Pese tener más ASP las especies continúan desapareciendo y los ecosistemas continúan degradándose. Los parques no son una panacea y no pueden soslayar los graves problemas estructurales de pobreza, desigual distribución de tierras y recursos, corrupción y racismo ambiental, entre otros. Si hubiera cabida al respeto, no habría necesidad de las ASP como las tenemos hoy. Si bien el reto de ayer era comprender el funcionamiento de los ecosistemas, el de hoy es integrar a las comunidades en su gestión; un intento de ello son las Reservas de la Biosfera -si bien no todas funcionan bien.

Ante este panorama, nos preguntamos que habría pasado si alguien nos hubiese advertido y preguntado: ...De acuerdo a nuestra economía, nos interesa producir cada vez más riqueza para "luchar contra la pobreza", por lo que ocuparemos áreas que preserven las fuentes de materias primas, así que les ofrecemos el paquete de áreas protegidas y sus diversas categorías de manejo. ¿Lo quieren o desean inventar Uds. su propio sistema de conservación? La respuesta ideal habría sido preguntar: ¿Y por qué mejor no cambiar ese afán acumulativo desmedido o, es realmente necesario para llegarle a los sectores empobrecidos; no ven acaso que todo funciona en sistema y al 
final unos pedazos aquí y allá no servirán como reservas fuente de materias primas? Hoy sabemos que la pobreza no se redujo, sino al contrario. Y si bien las ASP cada vez tienen menos probabilidades de resguardar las materias primas, en el ínterin del colapso sirven como fuentes de acumulación de riquezas vía el turismo y otros usos. El que ASP restrictivas $^{7}$-como los "parques nacionales"- se establezcan en zonas rurales donde las personas no tienen suficiente información, formación, ni poder de decisión, es racismo ambiental. Parece absurdo que existan zonas destinadas a proteger a los recursos silvestres que se regeneran rápidamente y no para aquellos, como los hidrocarburos y minerales, que demoran miles de años en hacerlo. El problema de fondo no es en realidad la pobreza, sino la acumulación de riqueza en el imperante sistema económico actual. Siempre se hacen esfuerzos de mapeos de pobreza en relación con la biodiversidad y conservación; lo ideal sería incluir a la riqueza o las fuerzas que generan tal pobreza.

El desarrollo sustentable implica un cambio que nos permita perdurar y la conservación de la diversidad biológica y cultural el mantenimiento de la variación que sustenta nuestra imperdurabilidad (i.e. la diversidad). Así, en el caso de la conservación, dura lo que varía y en el del desarrollo, lo que varía dura. No puede existir diversidad sin cambio, lo mismo que no puede haber desarrollo sin conservación. Al fragmentarlos, el uno impide al otro. En Meralvis estamos conscientes de la importancia de las áreas naturales remanentes y de las ASP como una vía para conservar la diversidad natural del planeta. No obstante, no olvidamos la perspectiva bajo la cual la mayoría de los parques fueron creados, ni las verdaderas razones de la tremenda disminución de los recursos naturales, ni perdemos de vista que lo importante de conservar tal biodiversidad implica también la continuidad humana en la Tierra. Al nivel latinoamericano, la solución no es tener áreas silvestres intocables y escindidas del ser humano. Hay que idear un sistema de conservación endógeno, capaz de fusionar distintos tipos de saberes y tecnologías para catapultar investigaciones y acciones transdiciplinarias en una carrera contra el tiempo, lo cual no es nuevo. Llamados a la transdisciplinariedad y hacia una nueva ciencia para la vida ya se han hecho (Belsky 2002, Rosa y Machlis 2002), lo mismo que la necesidad de un nuevo orden económico mundial y una nueva estrategia para la conservación de la naturaleza (Fergusson y Lanz 2001, Gilman 2001).

Hoy, sabemos que las ASP de América Latina fueron creadas con gran auge en los 1970s y 1980s, al amparo de una conservación convencional que ve a la biodiversidad como mercancía, en su lugar necesitamos Áreas Silvestres Integrales Alternativas (ASIA) donde haya un uso perdurable de sus recursos. Hace tiempo se sabe que las ASP no funcionan como debieran y que la fragmentación y deforestación acarrean consecuencias que han puesto a correr a cientos de organizaciones, científicos y técnicos por doquier ideando corredores biológicos, enlaces de paisajes, gestión de ecosistemas de cuencas,

\footnotetext{
${ }^{7}$ No permiten el uso de los recursos en ellas, salvo colectas científicas a la cual no llaman "cacería".
} 
entre otras, para prevenirlas de impactos irreversibles. En Costa Rica las ASP funcionan como islas socio-ecológicas en un mar de monocultivos con pesticidas y conglomerados urbanos, de transformarse en ASIA estarían integradas con la gente y sus ciudades. Extrapolando a la región, varios millones más de pobres, junto con un incremento cada vez mayor en la capacidad acumulativa de un pequeño porcentaje de personas, aunados a una pérdida sin precedentes de nuestro capital natural (ni hablar del social), incidirá en la existencia misma de la humanidad y más. Afectaremos, quizá irreversiblemente, los sistemas del planeta. Por tales razones sugerimos considerar un enfoque alternativo por y para la gente.

\section{Para perdurar los recursos naturales no deben ser pesados vía la economía neoclásica convencional.}

En una somera revisión de seis ejes diagnóstico (I- Aire, II- Tierra, III- Fuego, IVAgua, V- Cultura y sociedad y VI- Tecnología e industria; Fig. 1) vimos que las respuestas de la sociedad costarricense a los problemas socio-ambientales que vivimos son incorrectas. Por ejemplo, aunque haya habido una pequeña recuperación de bosques en ciertos sectores del país desde fines de los 1990s, ésta ha ocurrido bajo condiciones que implican el surgimiento de otros problemas (Fig. 2). Es notable la reforestación con maderables no nativos y gran parte del pequeño aumento boscoso ha ocurrido bajo un sistema mixto o privado de protección para el turismo y/o liado al pago de servicios ambientales -que, de 1995 al 2004, aumentó la tenencia de tierras privadas de 10.000 a 500.000 hectáreas (elaboración propia). La exclusión por privatización con brechas socio-económicas podría generar que bajo escenarios de crisis petrolera, aun los conservacionistas excluidos no toleraran más y usaran sin reparo los recursos, aunque éstos estén protegidos o tengan dueño, sino otro necesitado igual lo haría.

Figura 1: Elementos de la CIA-Sur según la Estrella de David.

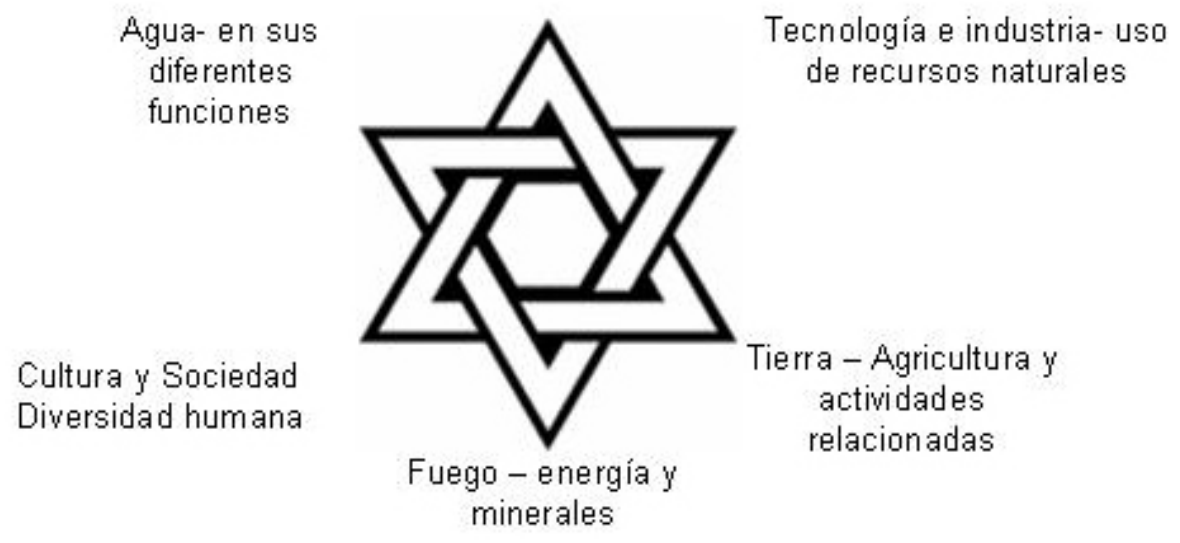


Figura 2: Representación cíclica del eje problemático "uso imperdurable de bosques de vida". RRNN: recursos naturales. Creación de un círculo vicioso donde se agrava el problema por soluciones paliativas; postergando su desenlace fatal. Imagen: Roberto Pérez Franco: http://rpf.com/index.php?leer=petolidib.

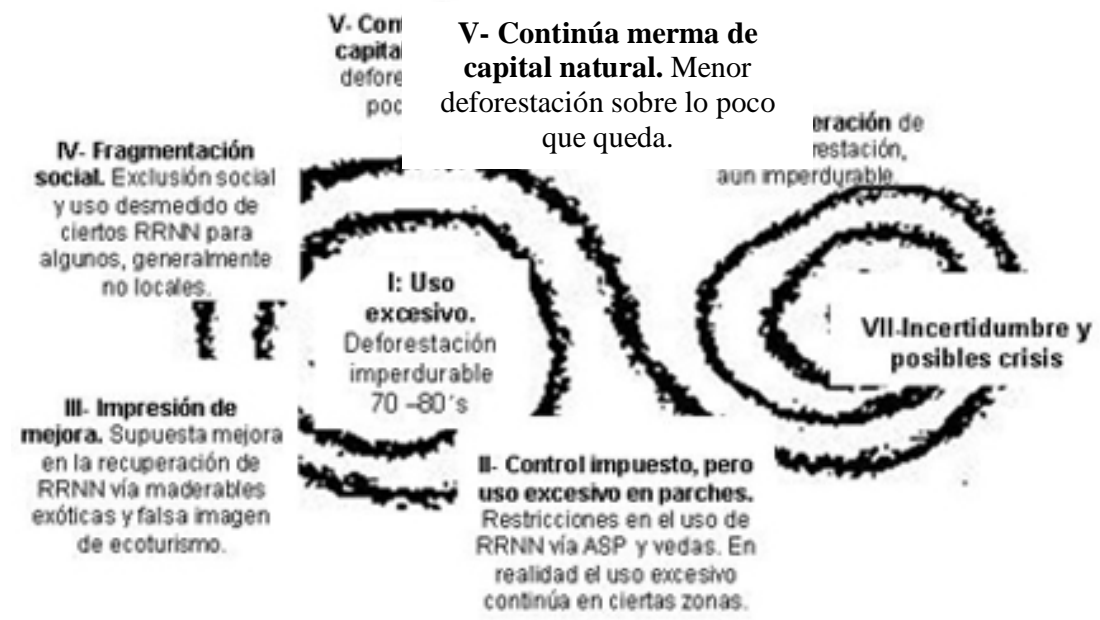

Al eliminar grandes extensiones de bosques no sólo hemos generado un cambio climático global, sino un incremento de la frecuencia e intensidad de desastres naturales. Aunque predicciones respetables al respecto indiquen que las perturbaciones serán cada vez mayores en frecuencia e intensidad, aún podemos implementar cambios que reviertan los efectos adversos que hemos creado, pero ocupamos del aval político-económico aunado al componente social. Aunque depende del tipo de bosque, en general un trozo de bosque bien manejado puede proporcionar suficientes recursos perdurables para cantidad de población (Diamond 2005), el problema es cómo y a qué tasa se usa al recurso bosque, además de a qué tasa lo regeneramos. Sin embargo, la información que se difunde en los medios es la incorrecta; por ejemplo se ha sugerido que el país posee mucho más bosque del que realmente tiene. No se quiere "ver" ni "dejar ver" que la deforestación continúa (Cuadro 2).

Cuadro 2. Porcentaje de bosque primario en Costa Rica por década (PEN 2006, Torrealba y Carbonell 2008).

\begin{tabular}{|l|l|}
\hline Año & Porcentaje de Bosque Primario \\
\hline 1960 & $56 \%$ \\
\hline 1970 & $51 \%$ \\
\hline 1980 & $29 \%$ \\
\hline 1990 & $22 \%$ \\
\hline 2000 & $16 \%$ \\
\hline
\end{tabular}


Si viéramos que todo parte de y termina en natura, entenderíamos que en lugar de pesar al ambiente por la economía, es la economía la que debiera ser pesada a través del ambiente pues este es el regidor. La economía debiera ser medida por natura, ya que ella es el capital madre y regidor. Por ello, urge que enfoques transdisciplinarios, como el marco que nos proporciona la economía ecológica, tomen ímpetu en investigaciones innovadoras que estén liberadas del instrumental ecológico y económico convencional. No es suficiente intentar complementar la economía con la ecología; para fusionarse hacen falta métodos que superen los enfoques existentes. Así, de acuerdo a nuestro enfoque de la CIA-Sur se necesita un nuevo tipo de economía aunado a áreas silvestre integrales alternativas que permitan un uso perdurable de los recursos de una forma socialmente equitativa.

\section{Para prevenir el colapso local en serie necesitamos visualizar nuestro Kaklavetzá particular}

Un aspecto común en Latinoamérica es que hay políticas nacionales que emulan un estilo de desarrollo incompatible con la perdurabilidad. Los indicios de que vamos por mal camino abundan. Comenzando con el abatimiento sistemático del agro desde hace unos 20 años y la eliminación del tren desde hace unos 15, aunados al fomento de furgones en las precarias carreteras y a la importación de alimentos; siguiendo, con el dengue en Guanacaste $^{8}$ en el 2007, el apagón nacional y los recortes periódicos de electricidad sufridos en el 2007, la contaminación de acuíferos y por primera vez el cierre de playas por tasa de coliformes fecales en el 2008, el nivel de polución ambiental y los problemas de tráfico vehicular en las zonas urbanas centrales y el incremento de eventos hidrometereológicos impactantes (su número promedio pasó de 168,6 entre 1970 y 2000; a 598,4 entre el 2001 y el 2005), implican que la ruta seguida está mal. En Costa Rica, al igual que en el resto de Latinoamérica, se sigue una historia de dominación y control al someter a grupos no-dominantes (Molina y Palmer 2000) por un fin acumulativo de unos pocos.

Por otra parte, no debemos perder de vista que más gente presionando sobre un mismo territorio pequeño, necesita de estrategias y políticas de sostenibilidad de los recursos, antes que otros lugares donde dispongan de más área y recursos. La Gran Área Metropolitana de Costa Rica posee más de 2.000.000 de habitantes en un territorio de apenas $1.778 \mathrm{Km}^{2}$ (al 2006 una densidad de 1.134 personas/ $\mathrm{Km}^{2}$ ) donde cada vez hay mayor segregación residencial sobre la base de la economía y más inseguridad ciudadana en un ecosistema urbano cada vez más deteriorado. El problema no es que el mundo mantenga un $20 \%$ rico entre norte y sur, ni dentro de cada país del sur (Hourtart 2006), sino

\footnotetext{
${ }^{8}$ Pobres prácticas sanitarias de lugareños vulnerables, más lluvias y descargas de lodos sépticos de hoteles para turismo extranjero en ciertas localidades han afectado la situación en esta zona seca, que pareciera no ser tan apta para el mosquito transmisor.
} 
que ése porcentaje está incrementando vertiginosamente su nivel económico y, por ende, el consumo de recursos y, por tanto, el daño medioambiental con repercusiones para toda la humanidad. Así, el problema crucial no es la cantidad de gente, sino la forma como usan los recursos. En Costa Rica, en 1988 el ingreso per cápita del trabajador independiente del quintil más rico era 10 veces el ingreso del quintil más pobre, en el 2004 esta relación fue de 38 veces (PEN 2006). En tan sólo seis años se triplicó el consumo de un sector de la población y esto, sin contar otros datos como la diferencia en cuanto a los salarios de los diputados; en lo que se ha llamado una economía de rapiña (Castro 2003).

La implementación de un desarrollo sustentable mal entendido, ha generado que en el 2007-más de 10 años después de haber iniciado como proyecto piloto de sustentabilidadCosta Rica no refleje un gran logro en materia del desarrollo sustentable aliado a la conservación de la biodiversidad. Por un lado, lo que hoy queda de representación silvestre menos perturbada, no sobrepasa el 25,7\% (ASP para el 2005) del territorio y, posiblemente, está por debajo de tal número, ya que los "parques" poseen complejas problemáticas y muestran diversos grados de perturbación y usos de su biodiversidad -sean estos legales o no. Por otro lado, en cuanto a desarrollo las brechas aumentan. Pese que el país gasta más en servicios sociales que otros países de la región y bastante más que el promedio de los demás países fuera de la región en etapas similares de desarrollo (entre un 1,5\% y un 1,8\% de su PIB); el nivel del gasto y el criterio de universalidad de muchos programas enmascara su eficacia real. Así, pese al crecimiento económico de inicios del siglo, muchos pobres y vulnerables no tienen entrada en los programas sociales, ni un acceso justo a los recursos que necesitan para sobrevivir (PEN 2006).

Adicionalmente, nuestros tomadores de decisión parecen permanecer centrados en problemas cortoplacistas y simplifican a niveles imposibles la cuestión socio-ambiental. Por ejemplo, pretender tener un balance neutro de Carbono (Ministerio de Relaciones Exteriores 2007) no sólo implica creer que podemos aislarnos del mundo, sino la corta visión de las conexiones y de los múltiples compuestos de los ciclos biogeoquímicos ligados a los sistemas humanos. Además, el problema no se reduce al Carbono unido a las ASP, antes el paradigma fue el Nitrógeno. La pasividad de los tomadores de decisión ante los problemas realmente importantes, así como sus respuestas erradas, han sido causa previa del colapso de grandes sociedades (Diamond 2005). Debemos integrar todas las actividades productivas y los asentamientos humanos, no sólo dentro de un ordenamiento territorial, ni un enfoque de cuencas o ecosistemas; sino dentro de una nueva ciencia transdisciplinar que nos permita un marco teórico capaz de hacerlo. De lograr encontrar nuestro kaklavetzá veríamos tales interrelaciones y buscaríamos soluciones. Kaklavetzá es una palabra de la etnia Cabécar de Costa Rica, que implica los poderes del viento, de la montaña, del agua, el fuego, los animales, la lluvia, las plantas, árboles y personas en un mismo territorio. Es una palabra holística que engloba el bienestar de los ecosistemas con el del ser humano, en una interrelación de reciprocidad y respeto. Mientras no 
encontremos el bienestar viendo como se degrada a pedazos nuestro capital social y ambiental aunque haya islas de ASP y burbujas de elevado consumo.

\section{El Sur Parasitado: Para sanar a América Latina debemos rescatar nuestro Duchicelam.}

Como la ciencia es ideológica y el conocimiento está fragmentado, no vemos el panorama completo, sino partes del mismo. Una visión reduccionista de las principales causas de la alarmante extinción de especies actual, es lo que Edgard O. Wilson ha denominado HIPPO. ${ }^{9}$ Fragmentación del hábitat, introducción de especies, crecimiento poblacional, contaminación y sobre-uso, afectan a las especies. Si tomamos al acrónimo HIPPO como directriz entonces propiciaríamos políticas proteccionistas. Sin embargo, al hacerlo cometemos grandes injusticias. Por ejemplo, en Costa Rica hay fuertes presiones por eliminar la cacería en todo el país, pero haciendo esto nosotros nos convertimos en exterminadores culturales. ¿Qué es un indígena sin bosque y sin caza? Acabamos su cultura al transplantar paradigmas de otros hacia ellos, como si ellos fueran responsables de la actual disminución de las poblaciones de fauna silvestre. Extinción de animales en el ápice de las cadenas tróficas como los grandes depredadores y deforestación van de la mano, sus causas están mucho más allá de lo meramente local. Pero estas mismas causas afectan las culturas también. Se fragmenta su cultura, se introducen visiones reduccionistas, crece la población al eliminar prácticas ancestrales que en algunos casos habían logrado mantener un grupo humano a una tasa de crecimiento acorde a la disponibilidad de sus recursos, la contaminación les llega a todos aunque no la generen todos en igual medida y se olvidan los principios culturales endógenos. Sin embargo, todas estas causas son en realidad efectos. Han sido empujadas por grandes fuerzas conductoras, donde las presiones político-económicas encabezan la lista. Poco se trabaja sobre estos factores indirectos, pero poderosos.

Es importante entender que hemos forjado una dinámica sistémica de enriquecimiento descomunal para algunos, a costa de la inequidad social que sufren muchos y grandemente subvencionados vía un enorme deterioro ecológico que nos afecta a todos (modificado de Quiroga 2001). Como sistema el mismo funciona hasta cierto punto. Políticamente lo llamamos "el sur parasitado". El parásito es un cazador perfecto, casi nunca mata a su presa, siempre logra vivir de él degradándolo pero no tanto como para menoscabar su propia existencia; el parásito logra esto en una estrategia que involucra múltiples sistemas (agua, tierra, animales, plantas, personas) a los niveles macro (visibles) y

\footnotetext{
${ }^{9} \mathrm{H}$ : pérdida y fragmentación de hábitat, I: introducción de especies, P: población excesiva, P: polución y O: olvido de los límites o sobre-uso de los recursos naturales vía la caza y la pesca, principalmente.
} 
micro (invisibles). Pareciera que nuestra gran riqueza y diversidad sólo ha servido para propiciar el crecimiento de otros a costas de nuestro propio "subdesarrollo". ¿Sería posible convertir un parásito comensal en un simbionte-mutualista?

Actualmente las fuerzas conductoras incluyen dos sistemas complejos en una dualidad contrapuesta. Por un lado, está duchicelam o el respeto que tenemos por la naturaleza e implica nuestro deber de salvaguardar nuestra cultura e historia de conservación. Un duchicelam es un símbolo mítico-religioso de la cohesión y anudamiento de la coyuntura socio-agrícola biológico-social que eterniza las estructuras de poder y los contenidos culturales de los pueblos agrícolas del sur. Es la historia misma testimonio de la confederación quitu-puruhuaya. Es un símbolo y expresión de las sociedades comunitarias y homogéneas, implica un continuador, un salvaguarda, un defensor y no solamente un mantaya o soberano. Es el Guía de un oleaje humano. Es el continuador de los ancestros y frente a la dependencia un duchicelam rescatará el árbol del ancestro pretendido por otros (Peñaherrera y Costales 1992).

Aunque se han hecho muchos estudios sobre los esfuerzos de integración de la gente a la conservación y su impacto en la sustentabilidad, no se ha verificado primero si la gente ya está integrada a la conservación ejerciendo un impacto positivo en la sustentabilidad. En América Latina muchas personas en el medio rural, incluso ante condiciones adversas, se mantienen firmes a sus principios y actúan de forma correcta. Personas de grupos no dominantes que son afectadas por las políticas ambientales y económicas convencionales y reduccionistas actuales, se mantienen con proyectos o ideas productivas compatibles y amigables con el ambiente que les permiten sobrevivir en lo económico. Tales personas, se mantienen luchando contra las corrientes de políticas que les desfavorecen e incitan a querer acumular más. Quienes ya están dentro de la conservación no necesitan ser integrados a ella, sino visibilizados.

De constituirnos seres reflexivos y proactivos podríamos bajar de pedestal a la tecnociencia (Zanotti 1998) y tenerla como socia y no como rectora, promoviendo así una transdisciplinariedad liberadora. Sólo así visibilizaremos nuestras instituciones latinas endógenas fomentadoras de la conservación que ya existen. Lo que hemos estado haciendo vía la conservación es una especie de rehabilitación ambiental, creando conectividad y vínculos enlazadores entre los fragmentos que han quedado. De haber usado nuestro profundo respeto por lo natural, habríamos modificado al medio inteligentemente, previendo la posibilidad de conexiones y vínculos vitales para el buen funcionamiento de los sistemas. Al deforestar o usar los recursos habríamos estado de una vez construyendo, no habríamos hecho un gran impacto destructivo. Sin embargo, todavía tenemos la posibilidad de revertir algunos aspectos y generar soluciones de perdurables.

En el otro lado, están las políticas ambientales Neoliberales que permiten un uso de 
los recursos naturales como si el ambiente fuera una despensa inacabable -las cuales pertenecen a las instituciones de la Gran Corporación Privada del sistema mundo de Wallerstein sensu De Venanzi (2001). ${ }^{10}$ Tales políticas nos impiden ver que desarrollo y conservación van de la mano. En pro de la opresión se hace que aprendamos a no aprender, debemos desaprender nuestro no aprendizaje para poder entender que conservación de la biodiversidad y el desarrollo sustentable, en sus acepciones depuradas, son uno mismo. Así, el parásito es la visión del mercado de la Gran Corporación Privada Perversa. ${ }^{11}$ En lugar de vivir independientemente con soberanía política, económica y alimentaria, hacemos que se consoliden identidades y etnicidades fijas a ciertos espacios y lugares, forjamos racismo ambiental y vemos como las áreas silvestres protegidas están en crisis debido a que son una estrategia de la globalización.

En América Latina vivimos lo que John Saxe ha llamado lo supraliminal, una crisis tan amplia que nuestra mente no alcanza a divisarla en su totalidad. Es la política y la economía lo que determina la distribución de los alimentos (Roberts y Thanos 2003), así los recursos naturales bajo una gestión diferente fomentaría la soberanía alimentaria. Después de la 2da Guerra Mundial la estrategia fue la Revolución Verde, la táctica fue una agricultura de uso masivo de pesticidas derivados del petróleo bajo el lema del miedo; más recientemente surgieron los transgénicos. Hoy día, los recursos naturales se usan vía las ASP a beneficio de otros, en parte vía el llamado ecoturismo -en realidad es green washing- y vía la prospección de la biodiversidad cuyo fin último es patentar la vida. La estrategia es, en el fondo, la misma: el uso de los recursos a un ritmo elevado de consumo, dependiendo del petróleo lo más posible y bajo el constante atemorizamiento de la mayoría, aunado a una serie de mensajes confusos, como medio muy rápido y efectivo de incremento del capital económico convencional y el poder de algunos. Tal sistema caerá cuando el capital radical que lo soporta, la diversidad biológico-cultural, colapse. El error de fondo es no considerar que los recursos, renovables al corto o al largo plazo, son finitos y dependen

\footnotetext{
${ }^{10}$ En la teoría del sistema-mundo el centro o el sol es la Gran Corporación Privada, alrededor del cual giran tres periferias planetarias. En la primera periferia, la más cercana al "sol", están los países que tradicionalmente formaron el centro anteriormente, tales como EEUU, Reino Unido, Japón y algunos países Europeos, los cuales importan materias primas y productos básicos y exportan residuos y tecnologías a las siguientes periferias. En la segunda periferia, están países como Taiwán, Malasia, Indonesia, Tailandia, Sri Lanka y Filipinas, entre otros, que exportan desechos a los países de las siguientes periferias y ciertos productos primarios a los países más cercanos al sol. En la tercera periferia, la más alejada del sol, estamos casi todos los países tropicales en vías de desarrollo de América y África que exportamos materias primas y alimentos a los países de las periferias más cercanas al sol, e importamos tecnología y productos acabados hechos con la misma materia prima que exportamos.

${ }^{11}$ Este mercado puede venir sea del Norte o de Asia, en todo caso se refiere al grupo privado internacional con poder político; en contraposición puede haber $-\mathrm{y}$, de hecho hay- grupos empresariales privados responsables que ya han empezado a buscar como tener una economía próspera y limitada, con responsabilidad social y natural.
} 
de una compleja red de interrelaciones cuya resiliencia no es eterna; cada vez será más caro obtenerlos de acuerdo al planteamiento de la economía Neoclásica y sus políticas Neoliberales y más difícil restaurarlos si residimos únicamente en la visión de la ecología y tecnociencia positivistas. Si fuéramos capaces de mantener el respeto por nuestro prójimo y por lo natural, ni siquiera necesitaríamos de áreas silvestres protegidas, no tendríamos la contaminación actual, no habríamos acelerado ni el calentamiento global, ni la desaparición de las especies; no habríamos llegado a crear y ser parte de un sistema tan perverso. En conclusión, para desparasitar al Sur se necesita rescatar y reconstruir nuestros valores propios de conservación, así como re-crear en cada sitio nuestras particulares CIA-Sur. 


\section{Bibliografía}

ALIDES (1997), Foros internos rurales: Compartiendo una iniciativa hacia la sostenibilidad. Cuaderno de trabajo. Alianza Centroamericana para el desarrollo sostenible. CINPE- UNA. Costa Rica.

Belsky, J. (2002), "Beyond the Natural Resources and Environmental Sociology Divide: Insights from a Transdisciplinary Perspective". Society and Natural Resources (Special Issue on Environmental Sociology and the Sociology of Natural Resources), 15(3): 269-280.

Botkin, D. y L. Talbot (1992), "Biological Diversity and Forests". En: S. Narendra (editora). Managing the World's Forests-Looking for balance between conservation and development (pp. 47-74). Iowa International Bank for reconstruction and development, Kendall/Hunt Publishers Co.

Buttel, F. (2002), "Environmental Sociology and the Sociology of Natural Resources: Institutional Historias and Intellectual Legacies". Society and Natural Resources (Special Issue on Environmental Sociology and the Sociology of Natural Resources), 15(3): 205-211

Callicott, J.B., L. Crowder y K. Mumford (1999), "Current normative concepts in conservation”. Conservation Biology, 13(1):22-35.

Capra, F. (2000), Ecology, community and Agriculture. California: Center for Ecoliteracy.

Carbonell, F. e I. Torrealba (2003), Informe final del proyecto "Una perspectiva integral para la conservación de los humedales del área de conservación Pacífico Central: El caso de Playa El Rey”. Maralvis, ONG-Meralvis y Área de Conservación del Pacífico Central. Costa Rica.

Idem (2005), Informe final del proyecto "Conservation of the Central American Tapir (Tapirus bairdii) and the La Amistad International Park, Costa Rica and Panama”. ONG Meralvis y CEPF/CI. Costa Rica.

Castro, G. (2003), De Civilización y Naturaleza: Notas para el debate sobre la historia ambiental latinoamericana. Simposio de Historia Ambiental del Centro de Estudios Latinoamericanos. Chile.

Chacón, M. (2003), Historia y Políticas Nacionales de Conservación. Ed.UNED. Costa Rica.

Clay, J. (1988), "Indigenous People and Tropical Forests: Models of Land Use and Management from Latin America". Cultural Survival Report, No. 27. 
Colchester, M. (2005), "Salvando la Naturaleza: Pueblos Indígenas, Áreas Protegidas y Conservación de la Biodiversidad". United Nations Research Institute for Social Development Discussion Paper, No. 55.

Dankelman, I. y V. Ramprasad (2000), "Biodiversidad en una perspectiva cultural". Compas, No. 2:4-6.

Davis, W. (2001), "Una luz al costado (fin) del mundo, conferencia". Documento del Centro cultural del BID, No. 41 (en: www.iadb.org/cultural/documents/encuentros/08130-41Davis.pdf)

De Venanzi, A. (2001), "Medioambiente y sistema mundo: La degradación ecológica y el envejecimiento de los negocios". Revista Venezolana de Economía y Ciencias Sociales (Tema Central: Ambiente, Economía y Desarrollo en el Contexto Latinoamericano), 7(1):133-148.

Diamond, J. (2005), Collapse: How Societes Choose to Fail or Succeed. Londres: Penguin Books. USA.

Fallas, J. (2003), "Bosques, calidad de datos y Estado de la Nación". Ambientico, 5(116):13.

Farrell, B. y L. Twining-Ward (2005), "Seven Steps Towards Sustainability: Tourism in the Context of New Knowledge". Journal of sustainable tourism, 13(2):109-122.

Fergusson, A. y R. Lanz (2001), "El desarrollo sustentable: ¿Paradigma de fin de siglo?". Revista Venezolana de Economía y Ciencias Sociales (Tema Central: Ambiente, Economía y Desarrollo en el Contexto Latinoamericano), 7(1):105-112.

Field, D. A. Luloff y R. Krannich (2002), "Revisiting the origins and distinctions between Natural Resource Sociology and Environmental Sociology". Society and Natural Resources (Special Issue on Environmental Sociology and the Sociology of Natural Resources), 15(3): 213-227.

Fournier, L. (2000), Desarrollo y perspectivas del movimiento conservacionista costarricense. San José de Costa Rica: Editorial de la Universidad de Costa Rica. Costa Rica.

Gilman, R. (2000), "Design for a Sustainable Economics". Dancing Toward the Future, No. 32 . Context Institute. (en: www.context.org/ICLIB/IC32/Gilman.htm)

Hinkelammert, F. (comp.), 1999. El Huracán de la globalización. Dep.. Ecuménico de Investigaciones. Costa Rica. 
Houtart, F. (2006), Movimientos sociales, cambio y esperanza mundial. Conferencia del Movimiento Pro Universidad Alternativa del Sur, Julio 27, 2006. Universidad Nacional, Instituto de Estudios Latinoamericanos. Costa Rica.

La Gaceta (1998), Decreto Ejecutivo No. 26827-PLAN (SINADES, Sistema Nacional de Desarrollo Sostenible) y Decreto Ejecutivo No. 26814 MP-PLAN-MINAE (CONADES, Consejo Nacional para el Desarrollo Sostenible). San José de Costa Rica: Poder Ejecutivo, Ministerio de Planificación y Ministerio de Ambiente y Energía. Costa Rica.

Leal, D. y O. Bonilla (2005), "Indígenas y campesinos americanos haciendo agricultura para la vida”. Ambientico, 29:23-31.

Lovelock, J. (2006), The Revenge of Gaia: Why the Earth is fighting back and how we can still save humanity. Londres: Penguin Books. USA.

Mann, C. (2006), 1491: New revelations of the Americas before Columbus. First Vintage Book Editions. Random House, USA.

McNeely, J., K. Miller, W. Reid, R. Mittermeier y T. Werner (1990), Conserving the World's Biological Diversity. Washington: UICN, BM, Instituto de Recursos Mundiales y WWF.

Mill, J. S. (1848), Principles of Political Economy. Ciudad México: Traducción del Fondo de Comercio Exterior, sobre la 7ma edición corregida por el autor de 1871.

Molina, I. y S. Palmer. (2000). The History of Costa Rica. Editorial de la Universidad de Costa Rica. Costa Rica.

Idem. (2005). Costa Rica: Del Siglo XX al XXI -Historia de una Sociedad. Editorial de la Universidad Estatal a Distancia. Costa Rica.

Monge-Nájera, J. (1999), The forgotten Banana Connection: Origin and evolution of Environmental Awareness in Costa Rica. Memoria de Congreso sobre Desarrollo Sostenible de la Universidad Estatal a Distancia, Editorial de la Universidad Estatal a Distancia. Costa Rica.

Muro, P. (2005), Investigación Interdisciplinaria para el Desarrollo Sostenible. Videoconferencia Agosto 5, 2005, de la Universidad Autónoma de Chapingo, México y Doctorado en Ciencias Naturales para el Desarrollo, Universidad Estatal a Distancia. Costa Rica.

Naredo, J. M. (1996), "Sobre el origen, el uso y el contenido del término sostenible". En: Ministerio de Obras Públicas, Transporte y Medioambiente de España. (Editor). La Construcción de la Ciudad Sostenible (en: habitat.aq.upm.es/cs/) 
Novo, M. (2005), Desarrollo Sostenible. Videoconferencia de Mayo 4, 2005, de la Universidad Estatal a Distancia de España y Doctorado en Ciencias Naturales para el Desarrollo. Costa Rica.

Obando, W. (2004), Diversidad de especies de plantas y vertebrados en Costa Rica. Información actualizada a Marzo del 2004. Heredia: Instituto Nacional de Biodiversidad. Costa Rica.

Palacio, G. (2001), En búsqueda de conceptos para una historiografía ambiental. Univ. Nacional/ICANH: 37-74. Colombia. Publicaciones CLACSO. Consultado el 10 de Marzo del 2008

http://www.clacso.org/wwwclacso/espanol/html/grupos/grupos/ecologia/germanpalac io.doc

Peñaherrera, P. y A. Costales (1992), La Real Familia Duchicela. Editorial Edym. España.

Pérez, S. y F. Protti. (1978). Comportamiento del Sector Forestal durante el período 1950-1977. Ministerio de Agricultura y Ganadería, Oficina de Planificación Sector Agrícola. Costa Rica.

PEN (2004), Programa Estado de la Nación para el Desarrollo Humano Sostenible, Informe No. 10. Programa Estado de la Nación para el Desarrollo Humano Sostenible. Costa Rica.

Idem. (2006), Programa Estado de la Nación para el Desarrollo Humano Sostenible, Informe No. 12. Programa Estado de la Nación para el Desarrollo Humano Sostenible. Costa Rica.

Quiroga, R. (2001), "Crecimiento económico, exclusión social e insustentabilidad ecológica: El caso de Chile 1974-1999”. Revista Venezolana de Economía y Ciencias Sociales (Tema Central: Ambiente, Economía y Desarrollo en el Contexto Latinoamericano), 7(1):161-179

Roberts, J. y N. Thanos. 2003. Trouble in paradise: Globalization and environmental crisis in Latin America Routledge editors. Reino Unido.

Rosa, E y G. Machlis (2002), "Its a bad thing to make one thing into two: Disciplinary distinctions as trained incapacities". Society and Natural Resources (Special Issue on Environmental Sociology and the Sociology of Natural Resources), 15(3): 251-261.

Scearce, D. y K. Fulton (2004), What if? - The art of scenario thinking for nonprofits. Global Business Network Community. Holanda. 
Skutnabb, T., L. Maffi y D. Harmon (2003), Sharing a world of difference: The Earth's Linguistic, Cultural and Biological Diversity. Amsterdam: Naciones Unidas (UNESCO), Terralingua y Fondo Mundial para la Naturaleza.

Solís, V. (2001), Biodiversidad y pobreza. En: F. Donato (editor). Memoria del II Congreso Nacional de Desarrollo Sostenible: Perspectivas hacia el Siglo XXI (pp. 44-55). Oficina de Planificación del Consejo Nacional de Rectores. Costa Rica.

Soulé, M. (1986), Conservation Biology: The science of Scarcity and Diversity. Massachusets: Sinauer Associate Publishers.

Stem, C., R. Margolius, N. Salasky. Y M. Brown (2005), "Monitoring and evaluation in conservation: A review of trends and approaches". Conservation Biology 19:295309.

Torrealba, I. y F. Carbonell (2002), Informe Final de Proyecto "Integrando la conservación y el manejo integrado de plagas en los Mariposarios del Área de Conservación Arenal (ACA)". Instituto Nacional de Biodiversidad, Área de Conservación Arenal, Maralvis 2000 y ONG Meralvis. Costa Rica.

Torrealba, I. y F. Carbonell (2008), "Cia-Sur: exclusión de la diversidad biocultural y riesgos en las áreas silvestres protegidas". Simposio Ecología, política y cultura en América Latina. II Congreso Latinoamericano de Antropología. Costa Rica.

Van Eijk, T. (1999), Science and Spirituality. Compas Newsletter. Feb 36-37.

Visser, L. (2003), Our common future: Development Sociology and the interaction between the social and the natural sciences. Wageningen University and Research Centre, Sociology of Rural Development, Inaugural Lecture on October 2. Holanda.

Wallerstein, I. (1996), "La estructuración capitalista y el sistema-mundo". Revista Venezolana de Economía y Ciencias Sociales, Vol. 3 (en ladb.unm.edu/econ/content/ecosoc/1996/january/estructuracion.htm)

WCMC (2006), Sitio URL de Centro de Monitoreo Mundial para la Conservación del Programa de Medioambiente de las Naciones Unidas (World Conservation Monitoring Centre, WCMC).

Woodhill, J. (2004), Facilitating complex multi-stakeholders processes - A social learning perspective. International Agricultural Centre. Holanda.

Zanotti, G. (1998). "La epistemología y sus consecuencias filosófico-políticas". Revista Libertas, $\mathrm{N}^{\circ} 29$ (en www.eseade.edu.ar/servicios/indice42.aso?ID=16). 